\title{
Motor unit identification in two neighboring recording positions of the human trapezius muscle during prolonged computer work
}

\author{
Journal Article \\ Author(s): \\ Zennaro, Daniel; Läubli, Thomas; Krueger, Helmut \\ Publication date: \\ 2003-08 \\ Permanent link: \\ https://doi.org/10.3929/ethz-b-000054018
}

Rights / license:

In Copyright - Non-Commercial Use Permitted

Originally published in:

European Journal of Applied Physiology 89(6), https://doi.org/10.1007/s00421-003-0821-7 


\section{Motor unit identification in two neighboring recording positions of the human trapezius muscle during prolonged computer work}

Accepted: 14 February 2003 / Published online: 24 April 2003

(C) Springer-Verlag 2003

\begin{abstract}
Work-related shoulder-neck pain is a major health risk in computer operators. To understand the physiological mechanisms behind the development of these disorders, EMG recordings of some minutes up to several hours must be accurately decomposed. For this reason we developed EMG-LODEC, an automatic decomposition software program, especially designed for multi-channel long-term recordings of signals detected during slight muscle movements. The subjects executed a 30-min computer task to simulate real work conditions while working at an ergonomically designed workstation. Six-channel intramuscular EMG signals were recorded from two positions of the upper trapezius muscle. The EMG signals were decomposed into individual motor unit action potential trains using EMGLODEC. The study design enabled us first to study the dependence of intramuscular analysis on the insertion points and second to test the accuracy of the decomposition technique under laboratory conditions during a real experiment. The two positions yielded 887 motor units -452 located in position 1 and 435 in position 2 . Although the numbers of detected action potentials were strongly correlated between the two insertion positions, different motor units were mostly recorded. In particular, the detection of continuously active motor units is specific for the selected insertion points and may not be representative of a muscle, not even for parts with common functions. The approach for the quantitative evaluation of the decomposition technique was to independently decompose two signals that were simultaneously detected by separate sets of wire electrodes placed close to each other in the muscle. Common trains discovered in each signal were compared for consistency. A cross-correlation analysis was performed to find
\end{abstract}

D. Zennaro $(\varangle) \cdot$ T. Läubli $\cdot$ H. Krueger Institute of Hygiene and Applied Physiology, Swiss Federal Institute of Technology Zurich, 8092 Zurich, Switzerland E-mail: daniel.zennaro@alumni.ethz.ch

Tel.: + 41-1-6324807

Fax: + 41-1-6321173 corresponding motor unit pairs that were concurrently active. Concurrently active motor units were found in six subjects. For these motor units the extent of simultaneous occurrence of motor unit action potentials between the two positions ranged from $23 \%$ to $78 \%$ depending on the distinction of the single motor units and the number of superimposed motor unit action potentials. High concordance was seen in 3 out of the 15 motor unit pairs. Based on the results, EMG-LODEC is capable of providing reliable decompositions with satisfying accuracy and reasonable processing time. EMGLODEC is suitable for the study of motor unit discharge patterns and recruitment order in subjects with and without musculoskeletal pain during long-term measurements to study work-related musculoskeletal disorders.

Keywords Accuracy - EMG signal decomposition · Long-term recordings $\cdot$ Wire electrodes $\cdot$ Work-related musculoskeletal disorders

\section{Introduction}

Information about the structural and functional characteristics of motor units (MUs) is highly relevant for understanding both neuromuscular control (Buchthal 1985; De Luca et al. 1982a) and neuromuscular disorders (De Luca et al. 1982b; Reiners et al. 1989; Stålberg et al. 1995). Intramuscular electromyography (EMG) is a well-established procedure for the study of muscle function. It has been shown to detect recruitment, de-recruitment, and the firing rate modulation of concurrently active MUs generated during excitation (Basmajan and De Luca 1985). Invasive techniques, e.g., employing needles or wires, have a high spatial resolution and are therefore suitable for the detection of single MU activity. In recent years many different techniques have been developed to decompose intramuscular EMG signals into basic units, either automatically or with 
some interaction from the operator (Gut and Moschytz 2000; Mambrito and De Luca 1984; McGill and Dorfman 1989; Ohlsen et al. 2001; Stashuk 1999). An excellent review of current and past activities in intramuscular EMG decomposition can be found in Stashuk (2001). Most automated EMG analysis techniques are developed for clinical short decomposition (about $10 \mathrm{~s}$ ), where constant muscle effort guarantees small shape-changes and regular activation patterns in the EMG signals and are applicable only to singlechannel measurements (Gut and Moschytz 2000; McGill and Dorfman 1989; Stashuk 1999). The problem is that to understand the physiological mechanisms behind the development of muscle fatigue, pain, and strain at lowintensity work, EMG recordings from a minute up to several hours duration must be accurately decomposed. For this reason we developed EMG-LODEC (ElectroMyoGram LOng-term DEComposition), an automatic decomposition software program (Zennaro et al. 2002, 2003). In contrast to existing decomposition methods, the decomposition software EMG-LODEC is especially designed for multi-channel long-term recordings of signals of slight muscle movements. The decomposition of these signals is highly dependent upon the valid identification of single motor unit action potentials (MUAPs). It is obvious that the performance of the decomposition technique affects both the statistics of the interpulse intervals and the MUAP waveform characteristics (Shiavi and Negin 1974). Thus, it is important to assess the accuracy of the decomposition technique. This is especially essential when using fine wire electrodes that have been developed for long-term measurements under dynamic muscle conditions. Needle electrodes are not well suited for studying recruitment patterns under dynamic conditions. Muscle movement induces position changes of the electrode in the muscle, which causes pain and affects the signal pick-up properties of the electrode (Basmajan and De Luca 1985). Wire electrodes, on the other hand, are fixed to muscle fibers due to the hook at the wire end, the movements are much less painful compared to using needle electrodes, and the baseline levels of the EMG signals are less influenced by the muscle movements (Kitahara et al. 2000). However, there is no control of how the wire tips are positioned relative to each other and to the muscle fibers. For this reason, the detected MUAPs are affected in an unknown way by the electrode technique and position. A very important aspect in the intramuscular EMG research field is, therefore, the evaluation of performance of decomposition techniques, a point not deeply investigated in the past. At present, the two most common methods for evaluating EMG signal decomposition results are: (1) the use of artificially generated signals with known features as a reference (Farina et al. 2001), and (2) the use of real measured signals decomposed by expert human operators as a reference (Mambrito and De Luca 1984; Stashuk 2001). However, it is clear that many physiological aspects in artificial signals are completely disregarded (primarily related to long-term signals). Furthermore, it has been shown that manual decomposition is subjective to a certain extent, even if performed by expert operators (Pilegaard et al. 2000).

In exploring the literature one finds that there are several recommendations for standard surface electrode position (Mathiasen et al. 1995). However, the optimal site of recording for the decomposition of intramuscular signals has yet to be investigated. There is a lack of information pertaining to the best site, especially in the trapezius muscle. A very close position would be optimal to have identical information at the MU level, but on the other hand it would not include any information on MU dimensions. Thus, based on practical reasons a distance of $2 \mathrm{~cm}$ was chosen in our experimental study design.

The purpose of this study was to assess the accuracy of decomposition results using multi-channel wires in two different recording positions of the upper trapezius muscle during prolonged computer work under laboratory conditions. Furthermore, the study design enabled us to study the dependence of intramuscular analysis on the insertion points.

\section{Methods}

Subjects

In total, 38 subjects ( 20 women and 18 men), ranging from 19 to 47 years of age [28.5 (6.2) SD], participated in the study. All subjects completed the standardized Nordic questionnaire (Kourinka et al. 1987) to obtain information about musculoskeletal disorders. Of the 38 subjects, 15 reported no pain - no more than 7 days with pain in the neck, shoulders, arms, wrist or fingers during the past 12 months. The remaining 23 suffered from musculoskeletal pain in the neck and/or shoulder regions. Approval was obtained from the ethical committee of the University Hospital of Zurich, and subjects gave their informed consent before participation.

\section{Experimental procedure}

The subject sat comfortably on a chair with back support. The chair was adjusted to enable the subjects to sit with their thighs horizontal and their knee joint at right angles. The desk was adjusted to a height such that, with relaxed shoulders, the upper arms were vertical and the forearms could be placed horizontally and parallel to the desk surface to support a relaxed position. The subjects executed an interactive computer-learning program [ErgoLight, (Guttormsen et al. 2000)] that was mainly mouse driven and lasted $30 \mathrm{~min}$. The task consisted of pointing the cursor at a target, activating it by clicking the mouse button, and then moving and rotating the target until an optimal position was obtained.

\section{Experimental arrangement}

Intramuscular EMG signals were recorded from two locations in the trapezius muscle with electrodes that consisted of four fine, Teflon insulated, stainless steel wires with a diameter of $80 \mu \mathrm{m}$ and length of $15 \mathrm{~cm}$ (Science Products, Hofheim Germany). The sterilized wires were inserted in a $26 \mathrm{G}$ hypodermic needle and twisted at the needle tip to ensure stable fixing within the muscle. The first four-wire electrode was inserted $2 \mathrm{~cm}$ medial and $1 \mathrm{~cm}$ above the midpoint between the $\mathrm{C} 7$ spinous process and the acromion (position 1). The second electrode was inserted $2 \mathrm{~cm}$ caudal to the 


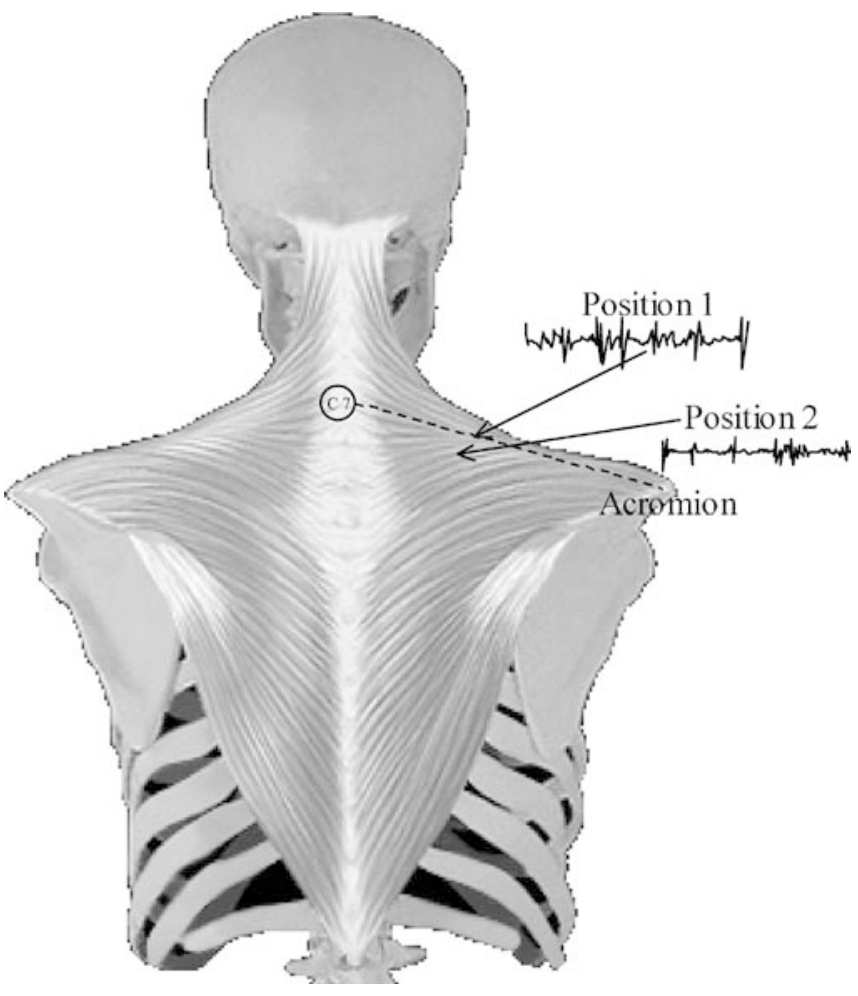

Fig. 1 Schematic description of the insertion points of the wires in the upper trapezius muscle

first one (position 2) (Fig. 1). The insertion angle was approximately $45^{\circ}$ and the insertion depth around $15 \mathrm{~mm}$ (Basmajan and De Luca 1985). The interelectrode distance was approximately $0.5-$ $3 \mathrm{~mm}$. The needle was removed, leaving the electrode inside the muscles. The wires were then fixed to the skin and connected to a differential amplifier (Dantec Keypoint) equipped with an electrically isolated preamplifier. The reference electrode was attached on the $\mathrm{C} 7$ spinous process. Using one wire as the common connector, two three-channel recordings were achieved. An anti-aliasing lowpass filter $(-100 \mathrm{~dB} /$ oct, cut-off frequency at $3 \mathrm{kHz})$ was applied to the signal. The signals were sampled at $20 \mathrm{kHz}$ and stored in binary format on a hard disk.

\section{Data analysis and statistics}

The stored intramuscular EMG signals were decomposed into individual MUAP trains using the automatic decomposition software EMG-LODEC, as reported in detail by Zennaro et al. (2002, 2003). The program uses wavelet coefficients, template matching, template updating and superposition resolution to identify the individual firing times of the MUs. The identification of specific MUs was performed simultaneously for three channels without operator interaction.

EMG activity was quantified with three outcome variables: (1) number of MUs: number of detectable MUs in the vicinity of the inserted wires, (2) number of MUAPs: sum of identified action potentials of all detected MUs, and (3) mean duration of MU activity: computation of all inter-spike intervals of MU " $i$ ". Those exceeding $1 \mathrm{~s}$ were defined as breaks and excluded from further calculation. The remaining inter-spike intervals were summed with the resulting period considered as the length of activity of a single MU. The MUs were divided into three different groups relating to their duration of activity: (1) sporadically (less than $50 \%$ of the experimental task), (2) intermittently (more than $50 \%$ and less then $90 \%$ of the experimental task) and (3) continuously active (more than $90 \%$ of the experimental task).
The reliability of the three outcome EMG variables between the two positions was elucidated by the calculation of the intraclass correlation coefficients (ICC coefficients) (Karras 1997).

\section{Method to test accuracy and reliability}

To test the accuracy of the decomposition program, artificially generated signals with known features were used as a reference (Zennaro et al. 2002). For details on the procedure for obtaining artificially generated intramuscular EMG signals, refer to Farina et al. (2001). The achieved accuracy of the decomposition program ranged from $92 \%$ to $100 \%$, depending on the complexity of the signals (e.g. the number of superimposed MUs). Those results could lead to the assumption that such an accurate decomposition generally results in reliable decomposition, but real signals are characterized by a high degree of variability and unpredictability. The decomposition program always represents a compromise between accuracy and the number of non-detected MUAPs. To make statements about the reliability and accuracy of real measured EMG signals, it is not sufficient to evaluate only the performance of decomposition techniques. The recording techniques also influence the acquired signals. The muscle force and the electrode type and positioning all affect the complexity and accuracy of identification. Thus, the reliability and accuracy of the decomposition program on real EMG signals was tested in the following way: the long-term recordings obtained from the two different positions (positions 1 and 2) were recorded simultaneously and decomposed. To detect the presence of MUAPs from the same MU in the two different positions, a cross-correlation analysis of the firing time intervals was performed (Basmajan and De Luca 1985). Cross-interval histograms were constructed from pairs of MUs by calculating the nearest forward and backward firing recurrence times of the alternate MU with respect to each firing of a reference MU. Figure 2 shows how we calculated the forward and backward-firing recurrence times. The recurrence times were then used to increment the bins representing the corresponding latencies in the crossinterval histogram. A bin width of $1 \mathrm{~ms}$ was used. If there is no relationship among the firing times of the MUs, the cross-interval histograms appear as a uniform distribution function. The probability of an occurrence is equal in all bins. The existence of peaks in the cross-interval histograms indicates the presence of interdependence. To systematically assess the existence of MU relationships

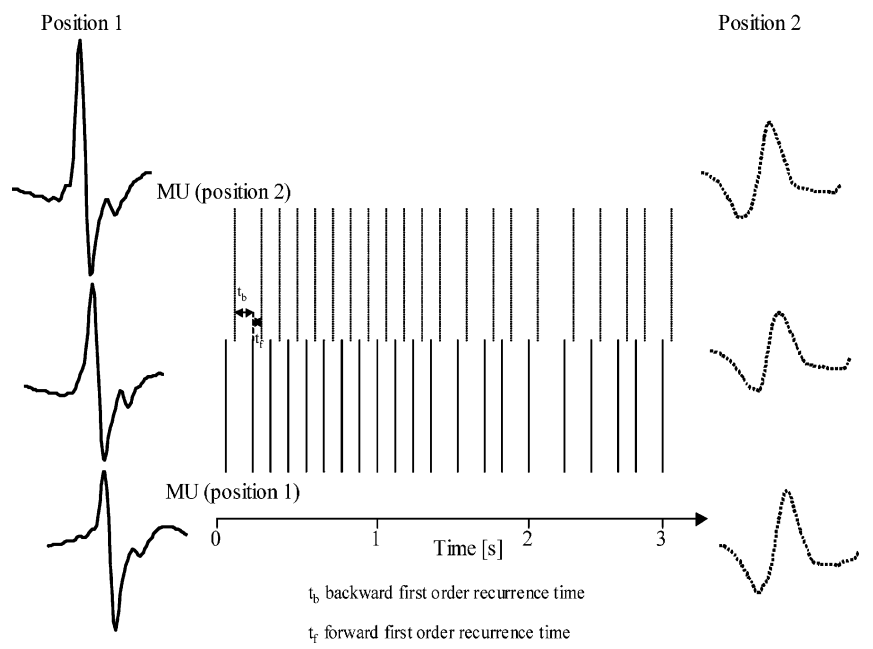

Fig. 2 Description of the forward and backward first-order recurrence times. Each motor unit action potentials $(M U A P)$ of the individual motor unit $(M U)$ is represented by vertical bars. The left hand side figures present the waveforms of the reference MU in position 1 for the three channels. The left right hand side figures illustrate the waveforms of the same MU in position 2 
and ensure that the peak is not an artifact of the random nature of the interfering intervals, the number of occurrences in a bin must be greater than the mean value at a $95 \%$ confidence level. The confidence level was calculated on the basis of the mean value of number of occurrences. This means that the number of occurrences must be greater than $20 \%$ of the number of MUAPs in position 1 or position 2. For each peak, the peak amplitude and the coincidence parameter were calculated. The peak amplitudes were defined as the number of occurrences in the bin within $\pm 2 \mathrm{~ms}$ from the bin with the highest number of occurrences (near 0 time delay). The coincidence parameter represents the extent of simultaneous occurrence of MUAPs between the two positions. This parameter was expressed as the percentage of the peak amplitude divided by the corresponding average value of detected MUAPs of positions 1 and 2 .

\section{Results}

For the data analysis, we used a $800 \mathrm{MHz}$ Pentium desktop computer. The analysis of the EMG signals takes between 30 and 120 min, depending on the MUAPs activity of the signals.

Motor unit activity in the neighboring recording positions

The decomposition program yielded 887 MUs - 452 located in position 1 and 435 in position 2 . The range of detected MUAPs was between 5 and 107,490 MUAPs. The mean firing rates varied between 7 and $30 \mathrm{~Hz}$. The majority of MUs were active during only part of the experimental session. Fifteen MUs were active during more than $90 \%$ of the experimental task (range of MU duration: 1406-1800 s). The intraclass correlation coefficient for the number of MUAPs between the two positions was high (ICC coefficient $=0.85$ ), as shown in Fig. 3. A weak correlation was found for the number of detected MUs between the two positions (ICC coefficient $=0.27$ ) (Fig. 3). The correlation for the number of continuously active MUs was also moderate (ICC coefficient $=0.51$ ).

Accuracy of the decomposition results (concurrently active MUs)

Concurrently active MUs were found in six subjects. The occurrences of the peaks occur within $\pm 2 \mathrm{~ms}$ of zero time latency between the discharges. The highest peaks were centered around zero time delay $[0.21(0.56) \mathrm{ms}]$ for all the simultaneously active MUs investigated. This indicates that the relative time delay between two MUs is due to the relative difference in the geometry and location of the wires and the conduction velocity of the axons innervating the MUs [mean muscle fiber conduction velocity in the trapezius muscle is approximately $4 \mathrm{~m} / \mathrm{s}=4 \mathrm{~mm} / \mathrm{ms}$ (Farina et al. 2002), i.e., time delay should be less than a few milliseconds, because the insertion points are oriented perpendicular to the fiber direction].
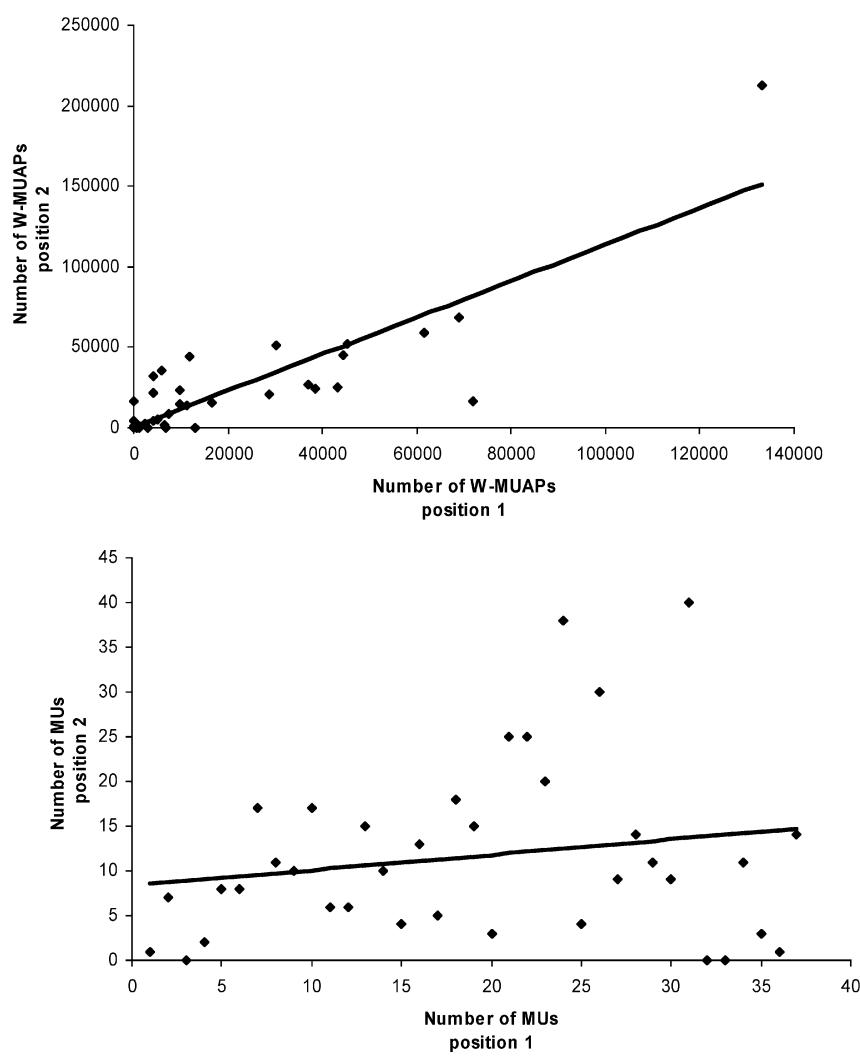

Fig. 3 The correlation of the number of MUAPs and number of MUs between the two positions. The correlation for the number of MUAPs between the two positions is high $($ ICC coefficients $=0.85)$, meanwhile a weak correlation is found for the number of MUs $($ ICC coefficients $=0.27)$

Figures 4 and 5 showed the detailed firing behavior during the 30-min computer task in those six subjects, where simultaneously active MUs in positions 1and 2 were observed. Out of the 30 individual MUs of the concurrently active MUs, 14 were sporadically, 12 intermittently and 4 continuously active. Four MU pairs fired continuously in both positions during the 30-min computer task (range of MU duration: 1300 $1800 \mathrm{~s}$ ). The other $11 \mathrm{MU}$ pairs did not always fire continuously in both positions. The pairs had different recruitment and de-recruitment patterns. Some MUs of those pairs fired later or had some breaks during the computer task (Figs. 4 and 5, MUs with the asterisks). This is one reason why the percentage of concurrently active MUAPs was not $100 \%$, although clear evidence of a relationship between the MUs was found. Figure 6 illustrates the firing rates of an intermittently active MU pair and a continuously active MU pair. The firing rates were obtained by passing the time series of the interpulse intervals through a Hanning window and inverting the output (Basmajan and De Luca 1985). The firing rate in the two positions are nearly identical. Choosing parts of the 30-min recordings, the coincidence resulted in $100 \%$ agreement for the observed MU activity with a total of 2000 firings or more. Generally, the amplitude and signal-to-noise-ratio 

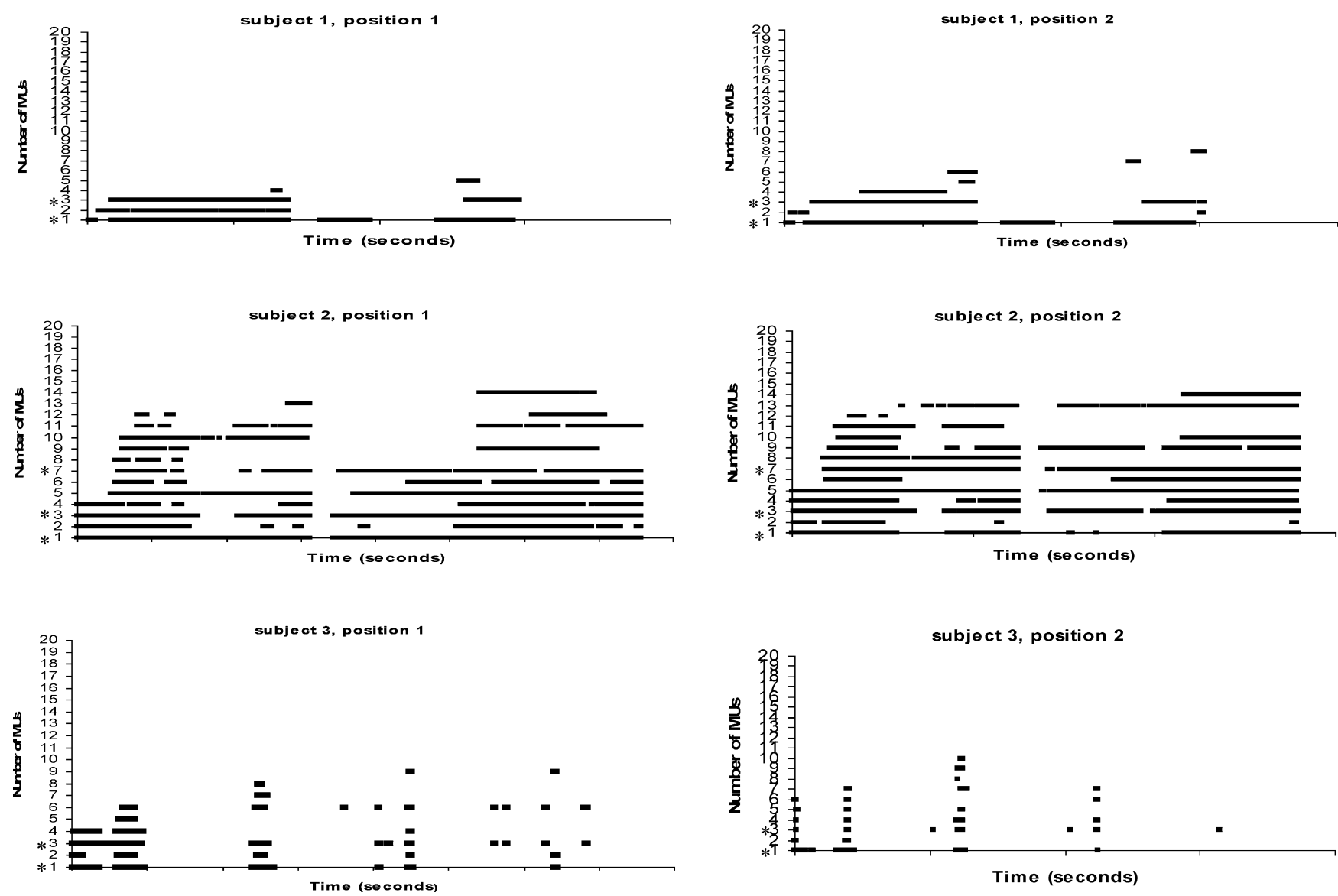

Fig. 4 MU activity bar plots from three subjects with concurrently active MUs in positions 1 and 2 (asterisks). Each horizontal bar represents the continuous activity of a single MU. Inter-firing intervals of more than $1 \mathrm{~s}$ are considered to be pauses. The recording time was $1800 \mathrm{~s}$

(SNR) are discriminators that describe the quality of intramuscular signals. The amplitudes of the 15 concurrently active MUs' MUAPs and the SNR ranged from 45 to $200 \mu \mathrm{V}$ and from 5 to $40 \mathrm{~dB}$, respectively [the SNR has been defined as the ratio of the peak energy and the signal energy between two adjacent peaks (Disselhorst-Klug et al. 1997)]. Neither parameter, however, was significantly correlated with a high simultaneous detection rate. Even very low amplitudes and SNRs resulted in a high coincidence parameter (the mean amplitude of MU 6 from Fig. 6 is $50 \mu \mathrm{V}$ with a SNR equal to $8 \mathrm{~dB}$ ).

The coincidence parameter for all 435 possible MU pairs ranged from 0 to $79 \%$ (Fig. 7). Defining the threshold by $20 \%$ concordance 15 MU pairs exceeded the threshold. For these MUs, the coincidence parameter ranged from 23 to $79 \%$. All the other MU pairs below this threshold fired simultaneously, but depending on the firing rate the action potentials were causally simultaneously active. They were only simultaneously active due to chance, which was checked by the inspection of the cross-interval histograms. In spite of the large distance between the two insertion points, we recorded

MUs that were simultaneously active in both positions (15 MU pairs). From this it follows that the electrical broadening of a few MUs is greater than $2 \mathrm{~cm}$. High concordance was seen in 3 out of the 15 MU pairs. The coincidence parameter was higher than $70 \%$. These MUs fired continuously during the 30-min computer task [MU duration: 1428 (174) s]. The other seven MUs did not always fire continuously, as shown in Figs. 4 and 5. The coincidence parameter lay between 23 to $65 \%$ depending on the distinction of the single MUs and the number of superimposed MUAPs. Two MU pairs reached high agreement for a relatively high number of MUAPs. Figure 8 shows the cross-interval histogram from these two MU pairs. The peaks indicating the relationship occurred within $1 \mathrm{~ms}$ on both sides of zero time delay. The number of firings ranged from 7805 to 19,378. In both cases the occurrences within $\pm 2 \mathrm{~ms}$ reached more than $70 \%$ concordance in both positions. Fifteen percent of the MUAPs of the 15 MU pairs were affected by observed variations in amplitude of the same MU ( $\pm 28 \%$ of the maximum peak-to-peak amplitude). Similar results were found in a previous study (Zennaro et al. 2002), where amplitude modulations in long-term recordings ( $10 \mathrm{~min}$ long) of more than $\pm 30 \%$ of the maximum peak-to-peak amplitude were observed. Although slow shape changes over time are correctly detected by the tracking algorithm, abrupt changes are often misclassified as newly recruited MUs. 

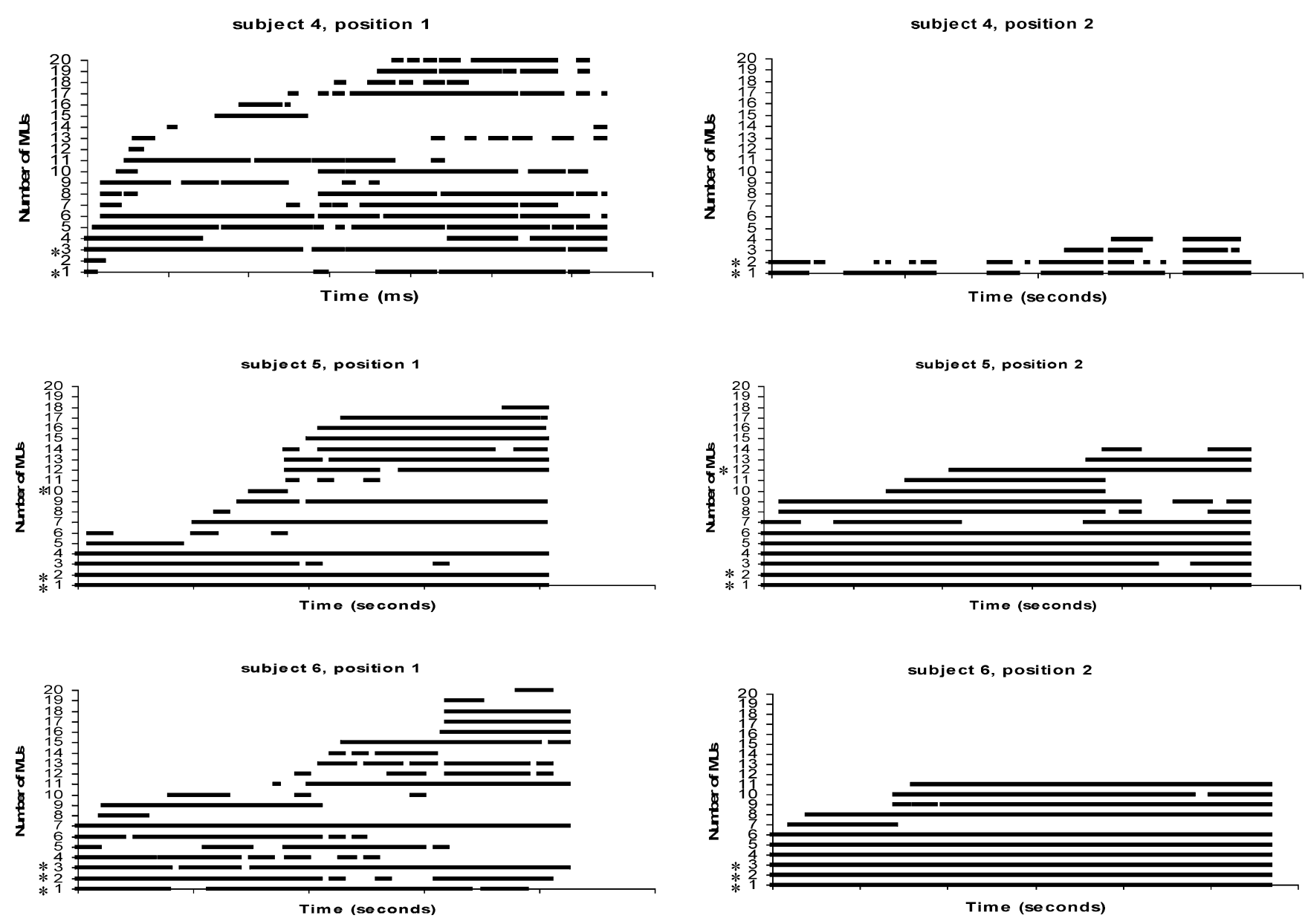

Fig. 5 MU activity bar plots from three subjects with concurrently active MUs in positions 1 and 2 (asterisks). Each horizontal bar represents the continuous activity of a single MU. Inter-firing intervals of more than $1 \mathrm{~s}$ are considered to be pauses. The recording time was $1800 \mathrm{~s}$

A MU showed a weak relationship with a second MU. The MU pair had 10,880 simultaneously detected MUAPs in position 1 and 2 and 1632 synchronously active firings to the MU with weak correlation. Scrutinizing these MUs, we could conclude that the two MUs in position 1 were the same MU. The firing rate of these MUs revealed clear evidence that these two MUs were actually one. Several factors accounted for the misclassifications. The waveforms of the MUAPs were very similar and the signal was heavily corrupted with noise, making it very difficult to decompose the signal. It should be noted that EMG-LODEC tends to overestimate the number of MU classes because it is important to discover all of the MUAPs trains significantly contributing to the signal and to try to minimize the number of erroneous classifications. In addition, it is easier to merge two MU trains into one than to split one train into two during analysis (Stashuk 2001).

A significant relationship for all MU pairs was found between the active MUs in position 1 and 2 and nonclassified segments in position 2 and 1, respectively (number of synchronously active firings was found to be

greater than $20 \%$ of the number of MUAPs in position 1 or 2). This indicates that some MUAPs were omitted by the decomposition program due to the MUAP changes caused by electrode or muscle movement, and eventually by changes of membrane properties. Furthermore, other factors may account for the difficulties of the decomposition: first, the low MUAP amplitude and second, the very low SNR (the signal is heavily overlapped with noise). The biggest reason for the misclassification was due to the superposition of two or more MUAPs. At least $60 \%$ of the non-classified segments are superimposed MUAPs, which the decomposition program could not decompose. The remaining non-classified segments were assigned to the previously described factors.

\section{Discussion}

The subjects executed a 30-min computer task to simulate real work conditions while working at an ergonomically designed workstation, ensuring a relaxed and comfortable body posture. The study design enabled us first to study the dependence of intramuscular analysis on the insertion points and second to test the accuracy and reliability of the decomposition technique under laboratory conditions during a real experiment. 
Fig. 6A, B The firing pattern of two MU pairs during the 30min computer task. A MU pair fired partly (A) and the other MU pair continuously (B)

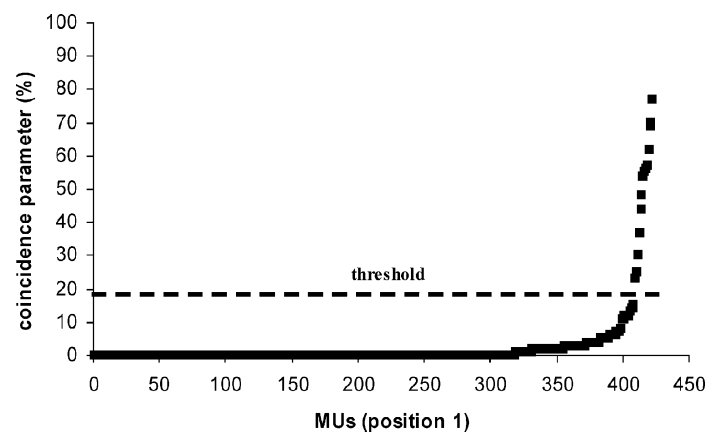

Fig. 7 The coincidence parameter for all 435 possible MU pairs. Defining the threshold by $20 \%$ concordance, 15 MU pairs exceeded the threshold (dashed line)

Motor unit activity in the neighboring insertion positions

The subjective effort in the neck-shoulder muscles during the experiment was minimal, which induced lowthreshold MUs. These low-threshold MUs may be very small, consisting of only a small number of fibers. Depending on their exact localization, they could easily be missed by the wires. The number of active MUs varied among the different localizations. The correlations were weak for the number of MUs and number of

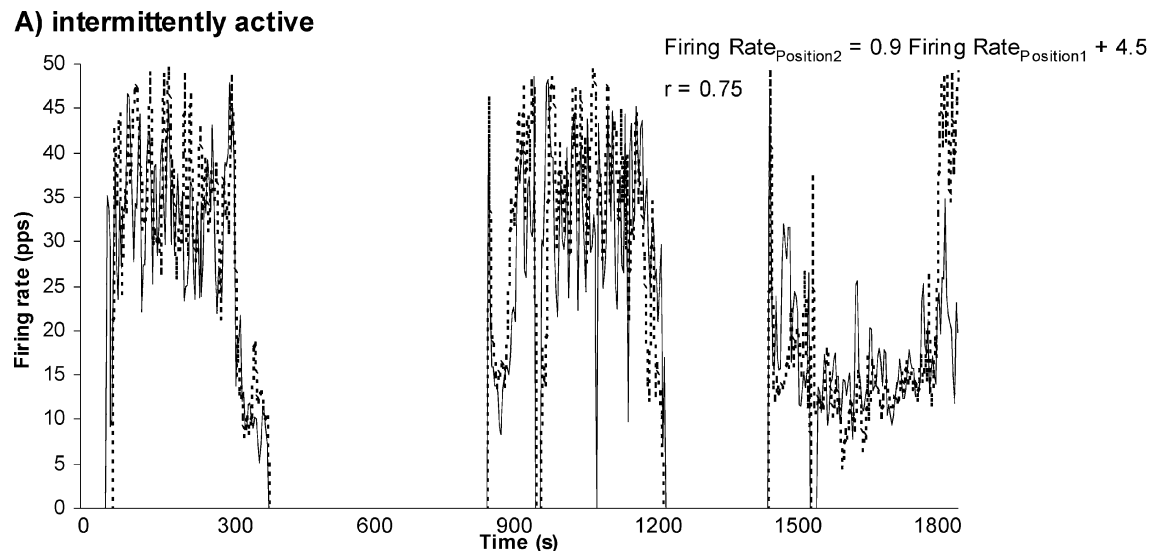

B) continuously active

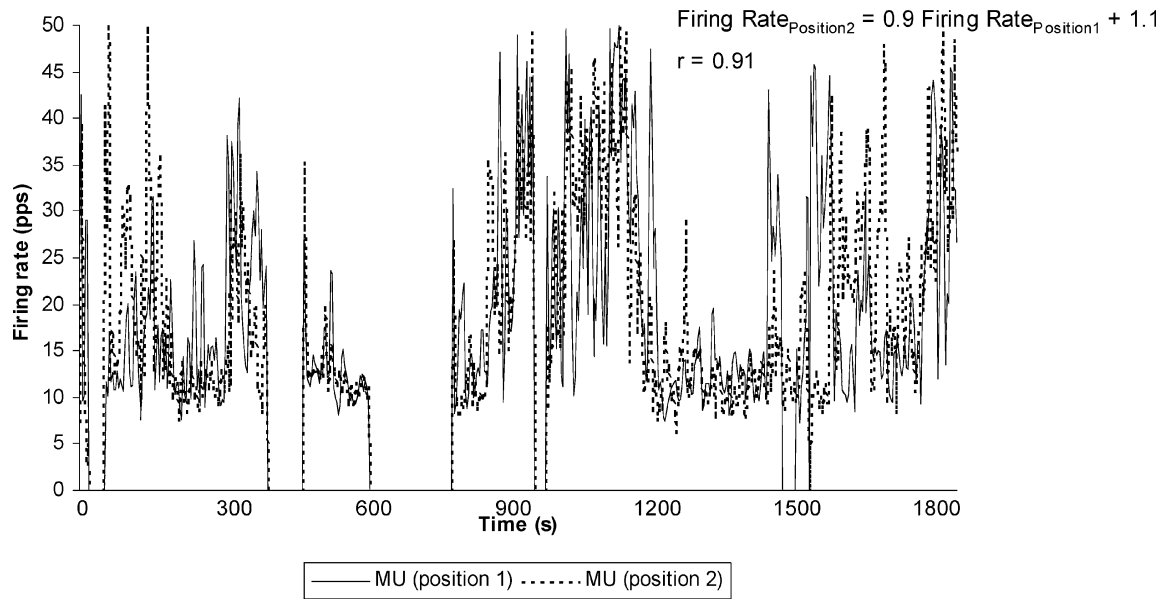

continuously active MUs between the two positions. The de-recruitment of MUs in one position does not predict the absence of MU activity in another location. However, the number of MUAPs showed a significant correlation between the two positions (Fig. 3). Different MUs were found within $2 \mathrm{~cm}$ of each other. Intramuscular EMG detection would, therefore, include a random selection of MUs. To overcome such restrictions the number of insertions and recordings should be high (at least in two different positions). Such an analysis can only be achieved with fully automated decomposition programs that allowed long-term analysis and rigorous control of the quality of the decomposition results. For particular applications, such as the decomposition of long-term recordings, the time required for the decomposition and the degree of interaction between the decomposition program and the operator (manual parts) should also be taken into account. Especially for longterm signals, where the amount of detected segments is high, it is impossible to decompose every signal manually.

Accuracy of the decomposition results

More and more research groups use intramuscular EMG recordings and there is a need for evaluating 

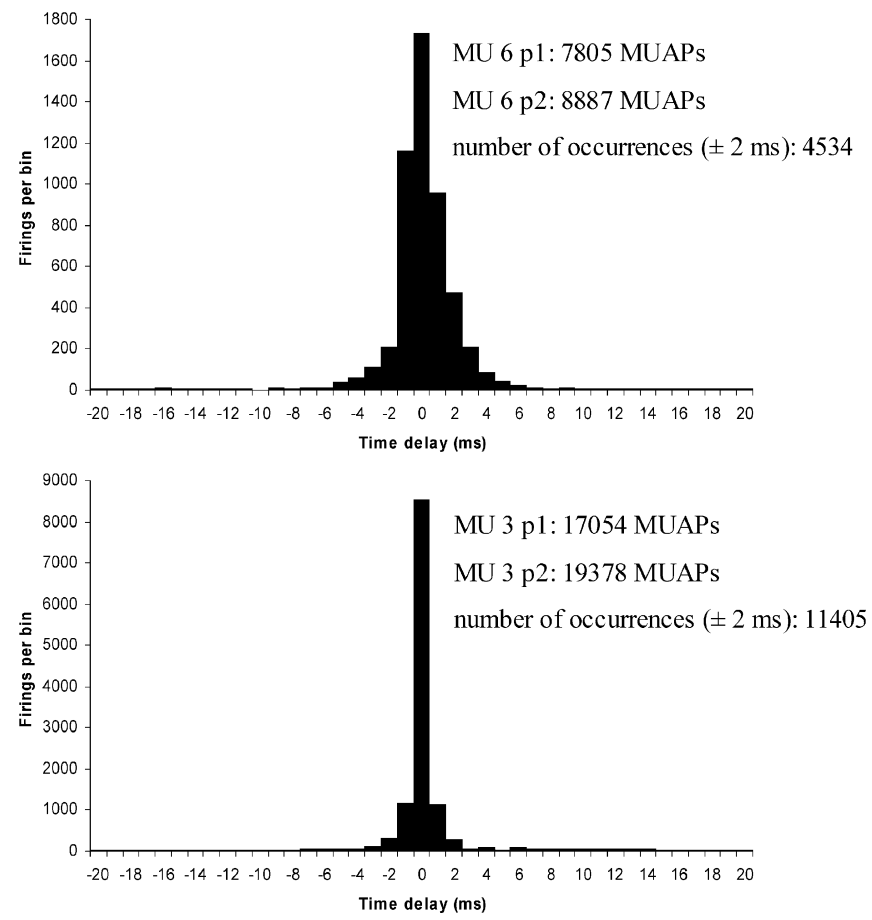

Fig. 8 An example of two cross-interval histograms from two different subjects during a 30-min computer task. For subject 1 the reference MU (position 1) fired 7805 times, and the appropriate MU in position 2, 8887. The number of occurrences is 4534. For subject 2 the MU in position 1 fired 17,054 times and the appropriate MU in position 219,378 . The number of occurrences is 11,405

decomposed signals with respect to reliability and accuracy, particularly for long-term recordings. Based on artificially generated signals, our decomposition software was capable of providing reliable decompositions with $100 \%$ accuracy (Zennaro et al. 2002). However, simulated signals cannot take into account all physiological aspects of the muscles. Some features of the synthetic signal may be quite different from those of the real signals or be completely disregarded. Furthermore, the consistency of the performance of a decomposition system across a variety of representative signals is very important (Stashuk 1999). The performance of the decomposition techniques has been tested for accuracy and reliability on real long-term EMG signals by comparing results relating the same MU simultaneously detected by separate electrodes and independently decomposed. This method provides an indirect measure of the accuracy and reliability of real data decomposition. That is because an undetected error in the results from one MUAP detection could occur only if a simultaneous error of the same kind is made in the decomposition of both recordings (Mambrito and De Luca 1984).

We tested the accuracy of the decomposition techniques during a real experiment under laboratory conditions and, therefore, we had to reach a compromise about where to insert the two electrodes. Due to the insertion of the second electrode in parallel fibers separated by $2 \mathrm{~cm}$ (perpendicular with regard to muscle fiber orientation), the expected number of concurrently active MUs in both positions was small. Henneman et al. (1965) stated that the distribution of the sizes of MUs and the MU territory diameters, respectively, resembles a Poisson distribution such that there are many more smaller MUs than larger ones. The muscle fibers of MUs are approximately randomly distributed throughout a subsection of the normal muscle and intermingled with fibers belonging to different MUs (Stålberg and Antoni 1983). A MU territory is considered as the circular crosssectional area in which all the fibers of a MU are located (Fuglevand et al. 1993; Stashuk 1993). The size of a MU's territory is determined by both the MU fiber density and the number of fibers belonging to the MU. Assuming that the distribution of muscle fiber diameters within a MU is constant across all sizes of MUs, it can be concluded that the size of a MU's territory is directly dependent on the size of the MU (Stashuk 1993). This in turn suggests that the more the larger MUs are near the two positions, the more likely it is that concurrently active MUs will be recorded. As a result of the muscle architecture, concurrently active MUAPs were observed when probably large MUs in the vicinity of the two electrodes were active.

The coincidence parameter ranged from 0 to $79 \%$, as shown in Fig. 7. It must be remembered that to detect MUAPs of maximum amplitude and sharpness, the electrodes should be close to active muscle fibers. That will, in turn, cause detected MUAPs to be more distinct, with respect to noise and sharpness. With wires, the muscle territories could not be explored to improve the signal quality. Thus, it is not surprising that the results of the two decompositions do not overlap $100 \%$ over the entire $30 \mathrm{~min}$. If we only took parts of the long-term signals, an accuracy of $100 \%$ was achieved (note the nearly identical firing rate of the MU pairs in Fig. 4 $(r=0.75$ and 0.91 , respectively)). Similar results were obtained by Mambrito and De Luca (1984) using needle electrodes. The comparison of the results from three different contractions with two common MUAPs train per contraction showed $100 \%$ agreement for a total of 1415 detections of the common MUAPs. In a highly standardized situation including slight isometric ramp contractions, Pilegaard et al. (2000) reported a coefficient of variation of $9 \%$ when describing the number of firings detected by two independent operators. Similar accuracy was achieved in the best of our recordings. In good conditions, long-term intramuscular recordings using wire electrodes may yield results of the same quality as achieved by needle recordings and computerassisted, visually controlled decomposition. Frequently used parameters for signal quality (MUAPs amplitude and SNR) do not allow one to estimate the accuracy and reliability of the decomposition results. Recordings that were achieved by using amplifiers of high quality, amplitude height and the SNR had minimal influence on the decomposition quality (Zennaro et al. 2002). It follows that the morphological parameters of the MUAPs 
and the characteristic parameters of the signals do not suffice to describe the quality of intramuscular EMG signals.

Difficulty arises when two or more discharges overlap in time to produce superposition. Resolving superpositions, i.e., determining the identities of the involved MUAPs and their precise time of occurrence, has been a major challenge in EMG decomposition. There are two fundamentally different methods. The first is the modeling approach (Stashuk 2001). It is based on synthesizing superimposed waveform models by adding up combinations of MUAP templates with relative time shifts. The second method is the peel-off or sequential approach (Stashuk 2001). It is based on matching MUAPs with the superimposed waveform. The latter method is used in our decomposition program, but the decomposition is limited to two overlapping MUAPs. Thus, to achieve higher concordance, the decomposition of superpositions must be improved. A reduction of the non-classified MUAPs would considerably increase the accuracy of the decomposition results. A decomposition program always represents a compromise between accuracy and the number of non detected MUAPs. In our opinion, it is more important that the decomposition results be accurate than to classify all MUAPs (e.g., superimposed MUAPs). The risk of missing MUAPs is higher than falsely identifying different MUAPs in the same category. This means that we may fail to identify a MUAP in a situation where it is active.

Another significant question, which is still not fully resolved, is if different MUs have been classified as the same MU. The MUAP waveforms of the MUs are very similar, especially when using wire electrodes. It is very difficult to completely eliminate this source of error, but the use of modeling could be helpful. Knowledge of the influence of wire movement and MUAP shape changes over time could be useful in determining if there are $\mathrm{MU}$ substitutions or shape changes in continuously active MUs.

The use of intramuscular techniques is useful whenever the activity of a single MU is of interest. Based on the results, EMG-LODEC is capable of providing reliable decompositions with satisfactory accuracy and reasonable processing time. EMG-LODEC is suitable for the study of MU discharge patterns and recruitment order in subjects with and without musculoskeletal pain during long-term measurements to study work-related musculoskeletal disorders.

Acknowledgements This work was supported by the Swiss National Science foundation (Project Number: 32-57163.99). The authors thank Dr. Denise Krebs for assistance with insertion of the electrode wires, and Dennis R. Ankrum and Kent Riopelle for valuable comments on this manuscript.

\section{References}

Basmajian JV, De Luca CJ (1985) Muscles alive. Williams and Wilkins, Baltimore, Md.
Buchthal F (1985) Electromyography for the clinician. In: Spittel JA Jr (ed) Clinical medicine. Harper-Row, Philadelphia, Pa.

De Luca CJ, LeFever RS, McCue MP, Xenakis AP (1982a) Behavior of human motor units in different muscles during linear varying contractions. J Physiol (Lond) 329:113-128

De Luca CJ, LeFever RS, McCue MP, Xenakis AP (1982b) Control scheme governing concurrently active human motor units during voluntary contractions. J Physiol (Lond) 329:129-142

Disselhorst-Klug C, Silny J, Rau G (1997) Improvement of spatial resolution in surface-EMG: a theoretical and experimental comparison of different spatial filters. IEEE Trans Biomed Eng 44 (No. 7):567-574

Farina D, Colombo R, Merletti R, Olsen HB (2001) Evaluation of intra-muscular EMG signal decomposition algorithms. J Electromyogr Kinesiol 11:175-187

Farina D, Fosci M, Merletti R (2002) Motor unit recruitment strategies investigated by surface EMG variables. J Appl Physiol 92:235-247

Fuglevand AJ, Winter DA, Patla AE (1993) Models of recruitment and rate coding organization in motor unit pools. J Neurophysiol 70:2470-2488

Gut R, Moschytz GS (2000) High-precision EMG signal decomposition using communication technique. IEEE Trans Signal Process 48 (No. 9):2487-2494

Guttormsen Schär S, Schluep S, Schierz C, Krueger H (2000) Interaction for computer-aided learning. Interactive Multimedia Electronic Journal of Computer-Enhanced Learning (http://www.wfu.edu/IMEJ/), Volume 2, No. 1

Henneman E, Somjen G, Carpenter DO (1965) Excitability and inhibitability of motorneurons of different sizes. J Neurophysiol 62:436-444

Karras DJ (1997) Statistical methodology: II. reliability and variability assessment in study design, part A. Acad Emerg Med 4:64-71

Kitahara T, Schnoz M, Läubli T, Wellig P, Krueger H (2000) Motor-unit activity in the trapezius muscle during rest, while inputting data, and during fast finger tapping. Eur $\mathbf{J}$ Appl Physiol 83:181-189

Kourinka I, Jonsson B, Kilbom A, Vinterberg H, Biering-Sørensen F, Andersson G, Jørgensen K (1987) Standardized Nordic questionnaire for the analysis of musculoskeletal symptoms. Appl Ergon 18:233-237

Mambrito B, De Luca CJ (1984) A technique for the detection, decomposition and analysis of the EMG signal. Electroencephalogr Clin Neurophysiol 58:175-188

Mathiasen SE, Winkel J, Hägg GM (1995) Normalization of surface EMG amplitude from upper trapezius muscle in ergonomic studies-a review. J Electromyogr Kinesiol 5:197-226

McGill KC, Dorfman LJ (1989) Automatic decomposition electromyography (ADEMG): methodologic and technical considerations. In: Desmedt JE (ed) Computer-aided electromyography and expert system. Elsevier, Amsterdam, The Netherlands, pp 91-101

Ohlsen HB, Christensen H, Søgaard K (2001) An analysis of motor unit firing pattern during sustained low force contraction in fatigued muscle. Acta Physiol Pharmacol Bulg 26

Pilegaard M, Jensen BR, Sjøgaard G, Søgaard K (2000) Consistency of motor-unit identification during force-varying static contractions. Eur J Appl Physiol 83:231-234

Reiners K, Herdmann J, Freud H (1989) Altered mechanisms of muscular force generation in lower motor neuron disease. Muscle Nerve 12:647-659

Shiavi R, Negin M (1974) The effect of measurements error on correlation estimates in spike-interval sequences. IEEE Trans Biomed Eng BME-9:374-378

Stålberg E, Antoni L (1983) Electrophysiological cross section of the motor unit . J Neurol Neurosurg Psychiatr 43:469-474

Stålberg E, Falck B, Sonoo M, Stahlberg S, Astrom M (1995) Multi MUP EMG analysis - a two years experience in daily clinical work. EEG Clin Neurophysiol 97:145-154

Stashuk DW (1993) Simulation of electromyographic signals. J Electromyogr Kinesiol 3:157-173 
Stashuk DW (1999) Decomposition and quantitative analysis of clinical electromyographic signals. Med Eng Phys 21 (No. 67):389-404

Stashuk DW (2001) EMG signal decomposition: how can it be accomplished and used? J Electromyogr Kinesiol 11:151-173

Zennaro D, Läubli T, Wellig P, Krebs D, Klipstein A, Krueger H (2002) A method to test reliability and accuracy of the decomposition of multi-channel long-term intramuscular EMG signal recordings. Int J Ind Erg 30/4-5:211-224
Zennaro D, Wellig P, Koch V M, Moschytz GS, Läubli T (2003) A Software package for the decomposition of long-term multichannel EMG signals using wavelet coefficients. IEEE Trans Biomed Eng 50/1:58-69 